\title{
Insulin, Ultralente
}

National Cancer Institute

\section{Source}

National Cancer Institute. Insulin, Ultralente. NCI Thesaurus. Code C29126.

A long-acting form of crystalline insulin often used in combination with a short-acting insulin in the treatment of diabetes mellitus. Administered once daily, this type of insulin may be derived from porcine, bovine, or recombinant sources. Endogenous human insulin, a pancreatic hormone composed of two polypeptide chains, is important for the normal metabolism of carbohydrates, proteins and fats; it has anabolic effects on many types of tissues. ( $\mathrm{NCl04})$ 